\title{
Cultural Isolation and Characteristics of the Blood Microbiome of Healthy Individuals
}

\author{
Stefan Panaiotov ${ }^{*}$, Georgi Filevski², Michele Equestre ${ }^{3}$, Elena Nikolova ${ }^{4}$, Reni Kalfin ${ }^{5}$ \\ ${ }^{1}$ National Center of Infectious and Parasitic Diseases, Sofia, Bulgaria \\ ${ }^{2}$ First Town's Hospital, Patriarch Eftimii 37, Sofia, Bulgaria \\ ${ }^{3}$ Istituto Superiore di Sanità, Viale Regina Elena 299, Rome, Italy \\ ${ }^{4}$ Institute of Experimental Morphology, Pathology and Anthropology, Bulgarian Academy of Sciences, Sofia, Bulgaria \\ ${ }^{5}$ Institute of Neurobiology, Bulgarian Academy of Sciences, Sofia, Bulgaria \\ Email: *spanaiotov@yahoo.com
}

How to cite this paper: Panaiotov, S., Filevski, G., Equestre, M., Nikolova, E. and Kalfin, R. (2018) Cultural Isolation and Characteristics of the Blood Microbiome of Healthy Individuals. Advances in Microbiology, 8, 406-421.

https://doi.org/10.4236/aim.2018.85027

Received: March 30, 2018

Accepted: May 28, 2018

Published: May 31, 2018

Copyright (c) 2018 by authors and Scientific Research Publishing Inc. This work is licensed under the Creative Commons Attribution International License (CC BY 4.0).

http://creativecommons.org/licenses/by/4.0/

\begin{abstract}
Background: On the analogy of the non-pathogenic microbiota found in oral cavity, skin and gastrointestinal tract, existence of blood microbiota was confirmed by DNA sequencing, but never deeply characterized. Hypothesis for the existence of dormant blood microbiota in healthy humans have been arisen and single species have been isolated. The aim of our study was to resuscitate and investigate the biodiversity of bacterial and fungal dormant blood microbiota in healthy individuals by blood culturing and NGS DNA sequencing. Results: Twenty eight blood samples of healthy individuals, seven for each blood type, were studied. Several culture media were tested. Blood microbiota resuscitation was performed in BHI broth supplemented with vitamin $\mathrm{K} 1 \mathrm{mg} / \mathrm{ml}, 2 \%$ sucrose, $0.25 \%$ sodium citrate and $0.2 \%$ yeastolate at $43^{\circ} \mathrm{C}$ for $72 \mathrm{~h}$. All tested blood samples were culture positive, as confirmed by Gram staining and TEM. TEM images demonstrated well defined cell structures. Analysis for bacterial and eukaryotic species was performed by $16 \mathrm{~S}$ rRNA and ITS2 targeted sequencing. The obtained sequences were clustered ( $\geq 97 \%$ identity) in Operational Taxonomic Units (OTUs). Among cultured and uncultured samples we identified OTUs similarity with 47 bacterial orders belonging to 15 phyla and 39 fungi orders blonging to 2 phyla. For the first time we demonstrated isolation and sequencing identification of fungal blood microbiota in healthy individuals. Blood-group differences were identified among the bacterial microbiome compositions. Conclusion: The dormant blood microbiome is innate of the healthy individuals. Interventional strategies to bind the host blood microbiome with the states of health and disease remain an unmet research goal.
\end{abstract}

\section{Keywords}

Blood Microbiota, Targeted Next Generation Sequencing, Operational 


\section{Introduction}

In the human and animal evolution many microbial species successfully adapted to the macroorganism. Most of them could not be cultured and are proven indirectly by DNA sequencing. On the bases of sampling 242 healthy adults at 18 different anatomical sites of the human body and sequencing the 16S rRNA genes the presence of 5177 microbial taxonomic profiles was proven, but only 800 of them could be cultured [1]. The observation of cell-free DNAemia is well described feature of the healthy blood [2] [3], but the presence of transient culturable blood microbiota in the blood of healthy individuals could also be supposed. We tested whether the presence of DNA in healthy blood is associated with DNAemia or it is due to existing blood microbiota. It is proven that in the blood of clinically healthy individuals microorganisms could persist for many years without causing illness. The most investigated examples being the latent tuberculosis.

Nevertheless the blood microbiome is still an enigma, its existence was proven during the last 50 years. Indirect evidences for existence of bacteria residing in erythrocytes have been predicted in the past by radiometric methods [4]. In 1969 Tedeschi et al. reported on incorporation of nucleosides in human erythrocyte attributed to the metabolic activity of mycoplasma or bacterial L-forms [4]. In 1977, Domingue and Schlegel identified in $7 \%$ of the blood specimens from supposedly healthy individuals novel bacterial structures [5]. In 1993 the Bulgarian scientist Emil Kalfin experimentally proved by culturing and electron microscopy that microorganisms are multiplying in the erythrocytes of healthy people [6]. Kalfin reported $100 \%$ positivity of the blood cultures. Subsequently several other authors questioned the existence of the blood microbiome or DNAemia in healthy individuals, Domingue (1977) [5], (1997) [7], Nikkari et al. (2001) [8], Mc Laughlin et al. (2002) [9], Moriyama et al. (2008) [10], Markova (2015) [11], Damgaard (2015) [12] Dimova et al. (2017) [13], Gosiewski et al. (2017) [2] and Kowarsky et al. (2017) [3]. The authors reported supporting electron microscopy, cultural and molecular data in favour of the existence of the blood microbiota in healthy individuals. Hypothesis on the bacterial structure of the blood microbiota in healthy humans have been arisen [5] [6] [12], but cultures for exhaustive microbiota analysis remain a target. Rich bacterial diversity in the blood of healthy individuals was confirmed by 16S rRNA genes sequencing [14] and total RNA sequencing [15]. In 2016 Paise et al. demonstrated that a diversified microbiome exists in healthy blood. Most of the blood bacterial DNA was found located in the BC (93.74\%), while RBCs contain more bacterial DNA (6.23\%) than the plasma (0.03\%) [14]. A significant number of bacterial species were also detected in the blood of healthy chickens [16] and cats 
by NGS analysis [17]. Resuscitation of dormant blood microbiota in healthy individuals has been tested and single bacterial species have been isolated on agar plates [12]. However, presence of eukaryotic microbial cells in the blood of healthy individuals has never been challenged. Goal of our study was to resuscitate the blood microbiota of healthy individuals and apply cultural, microscopic and NGS targeted sequencing methods for their characterization. Here, we demonstrate that bacteria and fungi constitute a rich microbial diversity in the blood of healthy humans by applying a successful culture resuscitation strategy, DNA isolation and NGS targeted sequencing analysis. First preliminary results of the study have been presented as poster at the ASM Microbe congress 2017 [18].

\section{Materials and Methods}

Ethical committee approval of the study (decision 38/14.07.2016) by the Institute of Neurobiology, Bulgarian Academy of Sciences (BAS) and individual written consent were obtained.

\subsection{Culturing}

Blood of 28 healthy volunteers was collected in Vacutainer tubes with $\mathrm{K}_{3}$ EDTA as anticoagulant (Vacutainer K3E, BD, USA), 7 samples per blood group. The blood samples were divided in two parts. One part for culturing $(3 \mathrm{~mL})$ and another part for direct DNA isolation $(7 \mathrm{~mL})$. All blood samples were tested for sterility by growing on Sabouraud and blood agar.

We applied a modified resuscitation strategy previously developed by Emil Kalfin [6]. Three $\mathrm{ml}$ of blood sample were added to $22 \mathrm{ml}$ of culture medium. Culturing was performed in sterile $50 \mathrm{ml}$ polypropylene Falcon tubes (Corning Inc, USA). The culture base medium was composed by Brain Hearth Infusion (BHI, Difco, USA) medium and $0.2 \%$ yeastolate (Difco) adjusted at $\mathrm{pH} 6.8$ and sterilized. Sterile $(\mathrm{D}+)$ sucrose at $10 \%$ final concentration and water-soluble form of vitamine $\mathrm{K}_{3}$ - menadione sodium bisulfite (Sigma-Aldrich, USA) in concentration of $1 \mathrm{mg} / \mathrm{ml}$ sterilized by filtration were added to the base medium. Resuscitation growth was induced at $43^{\circ} \mathrm{C}$ at $24 \mathrm{~h}$, but $72 \mathrm{~h}$ was found to be optimal time for growing blood microbiota in liquid cultures. The agar medium for subculturing contained 1.2\% Noble agar (Difco, BD, USA). Isolated blood microbiota was confirmed by Gram staining and 16S rRNA genes PCR analysis. Gram staining was directly applied on the cultured sample.

\subsection{Transmission Electron Microscopy (TEM)}

Blood samples for TEM were processed within 1 hour after collection. Sterile heparinized blood collected in Vacutainer tube (BD, USA) was diluted 1:3 with PBS pH 7.4 (Thermo Fisher Scientific, USA) and centrifuged at $400 \mathrm{~g}$ for $30 \mathrm{~min}$ on Histopaque-1077 (Sigma-Aldrich, USA) density gradient. The peripheral blood mononuclear cells were removed from the gradient, washed 3 times in 
PBS, then washed in RPMI 1640 (Sigma-Aldrich, USA) medium with 10\% FCS (Sigma-Aldrich, USA) and processed for TEM. The cells were resuspended in $3 \%$ low gelling temperature agarose (Sigma-Aldrich, USA) and put on ice. The solidified agarose was cut into $1 \mathrm{~mm}^{3}$ cubes, then fixed in $2.5 \%$ glutaraldehyde (Sigma-Aldrichm USA), postfixed in $1 \%$ osmium tetraoxide (Sigma-Aldrich, USA) and dehydrated in increasing concentrations of 30,50,70, 96\% of ethanol for $10 \mathrm{~min}$ each, $100 \%$ ethanol for $2 \times 20 \mathrm{~min}$ and propylene oxide for $2 \times 20$ min. Specimens were impregnated in propylene oxide: Durcupan ACM (Sigma-Aldrich, USA) 1:1 and embedded in Durcupan ACM. Polymerisation was carried out at $60^{\circ} \mathrm{C}$ for $18 \mathrm{~h}$ in an oven. The samples were thin cut to $2030 \mathrm{~nm}$ and observed under transmission electron microscope Opton EM 109 (Zeiss, Germany).

\subsection{Physico-Chemical Analysis and Radioresistance}

Disruption of cells with $0.1 \mathrm{~mm}$ glass beads with beat beater machine (Mini-beatbeater, Biospec Products, USA), treatment in microwave oven at 850 $\mathrm{W}$ and disintegration with ultrasound (MSE, UK) on ice was applyed. Integrity of the cells in fresh cultures was tested by treatments with $4 \mathrm{M}$ guanidine thiocianate, $10 \% \mathrm{NaOH}, 10 \% \mathrm{KOH}, 10 \% \mathrm{CH}_{3} \mathrm{COOH}, 10 \% \mathrm{HCl}$ and $10 \% \mathrm{H}_{2} \mathrm{SO}_{4}$ for 30 $\min$ at $37^{\circ} \mathrm{C}$.

To test the level of the ionizing radiation that the microbiota are able to withstand we applied gamma irradiation with ${ }^{60} \mathrm{Co}$ (Isledovatel MPX- $\gamma-25 \mathrm{M}$, TENEX, Moscow, RU) at 15, 20 and $25 \mathrm{kGy}$ to $5 \mathrm{ml}$ of blood from three healthy donors and erythrocyte concentrate of blood group $\mathrm{A}, \mathrm{Rh}(+)$. The erythrocyte concentrate was a sample produced by collecting blood from several blood donors at the National Center for Transfusiology and Hematology in Sofia, Bulgaria. In order to control the quality of irradiation two control blood samples were spiked with Candida guiliermondii and Candida albicans with $10^{9}$ cells. After irradiation with dose of $15 \mathrm{kGy}$, fungi inoculated control samples were tested for growth on Sabouraud agar. Control plates were negative after two weeks of culturing at $30^{\circ} \mathrm{C}$.

\subsection{DNA Isolation and Sequencing Analysis}

Seven commercial kits were tested for DNA isolation of the cultured blood microbiota: Tissue and cells genomic Prep kit and Blood genomic Prep kit, (GE, USA); Genomic DNA purification kit (Thermo Fisher Scientific, USA); QIAamp DNA isolation kit (Qiagen, Germany); NucleoSpin Soil and NucleoSpin DNA Stool kit (Macherey-Nagel, Germany); and RIBO-prep kit (Ecoli s.r.o., Slovakia). Most of the DNA extraction kits: Tissue and cells genomic Prep kit and Blood genomic Prep kit, (GE, USA); Genomic DNA purification kit, (Thermo Fisher Scientific, USA); QIAamp DNA isolation kit, (Qiagen, Germany) and the standard CTAB phenol/chlorophorm DNA extraction protocol were not successful for DNA extraction of the cultured blood microbiota. Successful DNA extrac- 
tions were obtained with NucleoSpin Soil and NucleoSpin DNA Stool kits (Macherey-Nagel, Germany) and RIBO-prep kit (Ecoli s.r.o., Slovakia) applying the following essential modifications: After $72 \mathrm{~h}$ of culturing the tubes were centrifuged at $5000 \mathrm{~g}$ for $15 \mathrm{~min}$. The pellet was washed by vortexing with $40 \mathrm{ml}$ of DNase I $1 \mathrm{U} / \mathrm{ml}$ (Thermo Fisher Scientific, USA) in $\mathrm{ddH}_{2} \mathrm{O}$ and incubated for $30 \mathrm{~min}$ at $37^{\circ} \mathrm{C}$ in order to eliminate residual DNA contaminations associated with lysed nuclear blood cells, DNA from the culture medium or DNA traces in water. Centrifugation and washings were repeated three times. These steps lyse the blood cells, wash away medium and human blood cells DNA/RNA contaminations. The cell pellet was resuspended in $2 \mathrm{ml}$ of TE buffer ( $\mathrm{pH} 7.5$ ). The same procedure was applied for direct microbiota isolation from whole blood. Briefly, $7 \mathrm{~mL}$ of blood were lysed by vortexing with $40 \mathrm{ml}$ of DNase I $1 \mathrm{U} / \mathrm{ml}$ in $\mathrm{ddH}_{2} \mathrm{O}$ and incubated for $30 \mathrm{~min}$ at $37^{\circ} \mathrm{C}$. After centrifugation at $5000 \mathrm{~g}$ for 15 min the same procedure was repeated three times. The pellet from the lysed whole blood was resuspended in $400 \mu \mathrm{l}$ of TE buffer. One hundred $\mu \mathrm{l}$ of cell suspension was processed according to the manufacturer instructions and modifications. The cell suspension was subjected eight times to freeze-thawing in liquid nitrogen and dry bath at $96^{\circ} \mathrm{C}$ for 10 min combined with vigorous vortexing or optional homogenization with ceramic/zirconium $0.6-0.8 \mathrm{~mm}$ beads on a bead beater for $5 \mathrm{~min}$ (Biospec Products, USA). The extracted DNA was resuspended in $100 \mu \mathrm{l}$ of TE and repurified by addition of $5 \mu \mathrm{l}$ of $20 \%$ Chelex 100 (Bio Rad, USA). Suspension was vigorously vortexed for $1 \mathrm{~min}$ and centrifuged for $10 \mathrm{~min}$ at $15.000 \times \mathrm{g}$. The supernatant, $\sim 95 \mu \mathrm{l}$ was recovered in a new tube without touching the Chelex 100 pellet. DNA typical yield was $>150 \mathrm{ng} / \mu \mathrm{l}$ with a $260 / 280$ $\mathrm{nm}$ ratio of $\sim 1.7$.

For DNA analysis we applied 16S rRNA genes and ITS2 targeted sequencing on Illumina MiSeq (Illumina Inc., USA). DNA sequencing was performed at IMGM Laboratories GmbH (Martinsried, Germany). For bacteria identification V3 - V4 hypervariable regions of the 16S rRNA genes were amplified with universal primers $314 \mathrm{~F}$ and $805 \mathrm{R}$. For fungi identification ITS2 region was amplified with universal primers ITS3 and ITS4. The obtained sequences were clustered ( $\geq 97 \%$ identity) into Operational Taxonomic Units (OTUs). OTUs were analyzed for sequence similarities against reference sequence databases; SILVA database for 16S rRNA gene (https://www.arb-silva.de/) and UNITE for ITS2 (https://unite.ut.ee/). Common sequences between the samples and the negative $\left(\mathrm{dH}_{2} 0\right)$ and culture medium DNA controls were excluded from analysis. These were considered as contaminants from reagents and culture medium. For taxonomic affiliation the interference created by the MiSeq sequencer as well as those created between samples was also considered.

\section{Results and Discussion}

All tested blood samples were culture positive, as confirmed by Gram staining and TEM (Figure 1). Optimal growing temperature was at $43^{\circ} \mathrm{C}$. At $37^{\circ} \mathrm{C}$ growth of the blood microbiota was suppressed. However, to our knowledge 


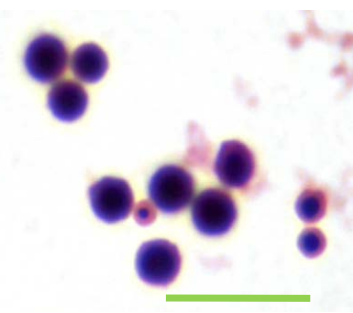

(a)

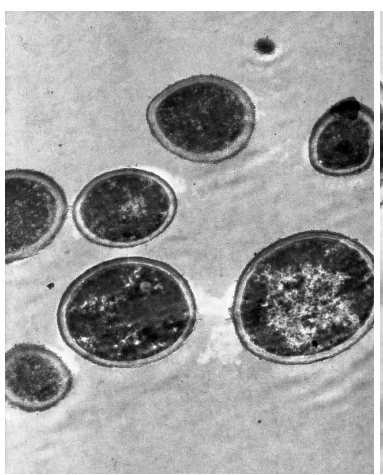

(d)

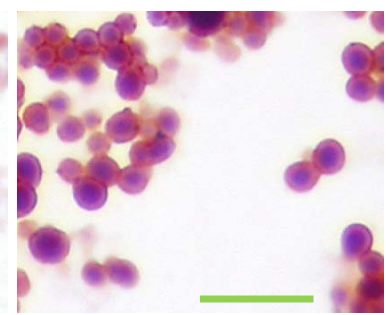

(b)

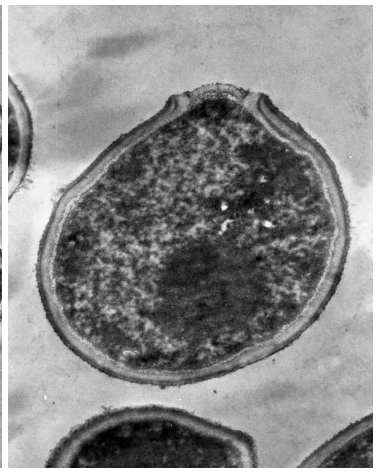

(e)

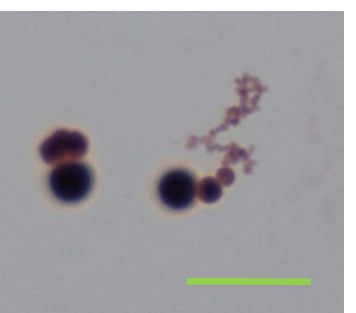

(c)

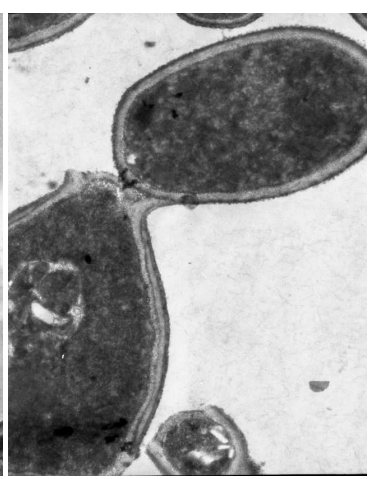

(f)

Figure 1. Microscopic examinations of blood microbiota. (a) Gram stained normal blood microbiota of cultured blood at $43^{\circ} \mathrm{C}$ for $48 \mathrm{~h}, 10 \times 100$. Several morphological forms could be observed; (b) "Dense body" of Gram positively stained microbial core structure surrounded by Gram negative coat. (b), (c) The "dense body" could divide by simple budding, and/or (c) produce in chains Gram negatively stained "primary cells". Green bar $10 \mu \mathrm{m}$; (d) (e), (f), TEM of blood microbiota in stages of maturation and division. The blood sample was processed within 1 hour after collection. Magnifications: Originally (d) $\times 7600$; (e) $\times 12700$; (f) $\times 12700$.

evidences completely lacking that microbiological isolation of the blood microbiome in healthy subjects is possible within 48 hours. The applied methodology of isolation in liquid culture media is technically affordable and repeatable, although we did not found appropriate conditions to maintain viable the blood microbiota in subcultures. In subcultures the blood microbiota revert to their dormant state. We did not identified the factors responsible of this process.

We observed several morphological forms of Gram stained blood microbiota, previously described by Domingue [5] [7] as "dense body" of Gram positively stained microbial core structure surrounded by Gram negative coat and chains of Gram negatively stained "primary cells" (Figure 1). TEM images demonstrated well defined cell structures (Figure 1). TEM and culture results confirmed that the blood microbiome represents viable structures rather than debris resulting from degradation of blood elements, such as lipids or haemoglobin complexes. We suppose that the "dense body" is a compact mass composed by "primary cells". Microscopic evidences are shown on Figure 3(c). Microscopic slides have poor stainability for DNA with Acridine Orange (AO). Impermeability is probably due to difficult stain penetration even after $15 \mathrm{~min}$ of staining in 1 $\mathrm{mg} / \mathrm{ml}$ of $\mathrm{AO}$.

After optimizing growth media and temperature conditions for liquid cultures 
next step was to develop agar media for isolation. Before subculturing on agar plates all liquid cultures were tested for contamination on Sabouraud and sheep blood agar. Cultures were negative for contaminations. Subculturing on agar was performed from $48 \mathrm{~h}$ liquid culture. Blood agar plates were prepared by adding to the optimized liquid medium 1.2\% Noble agar (Difco, BD, USA) and 10\% sheep blood. We found that sheep blood agar was not suitable for isolation of human blood microbiota. Growth was not visible with naked eye even after prolonged cultivation for more than two months. Isolation strategy was changed by preparing agar plates with homologous human blood. Agar plates prepared with $10 \%$ blood of the same individual used for inoculation of liquid culture gave partial growth. Plates were sealed in plastic bags to diminish drying of agar and culturing was performed at $43^{\circ} \mathrm{C}$. After 30 days of culturing brown colonies, with no odour and diameter of $0.5-1 \mathrm{~mm}$ were observed (Figure 2). Longer periods of culturing do not give increase in colony size, due to the fact that agar is drying and somehow growth seems inhibited or much slower. Agar plates with $10 \%, 20 \%$ and $80 \%$ of homologous blood were tested and there was no difference in growth efficiency. The bacterial colonies were confirmed visually and by Gram. Isolation of colonies on agar media of the blood microbiota from healthy individuals was partly successful and still challenging. Subcultures are unstable.

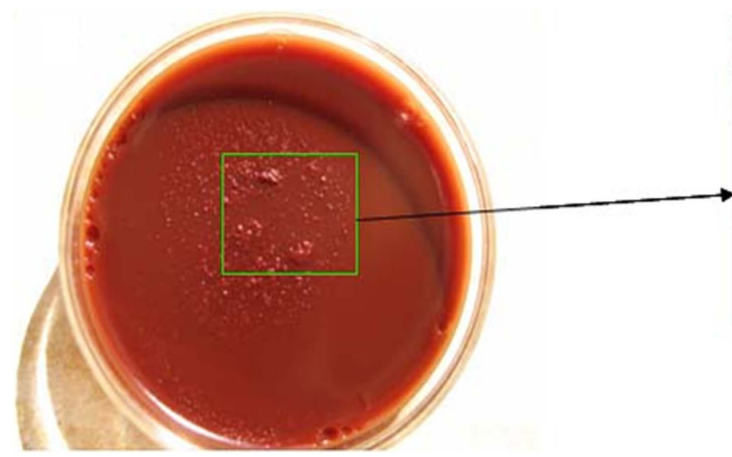

(a)

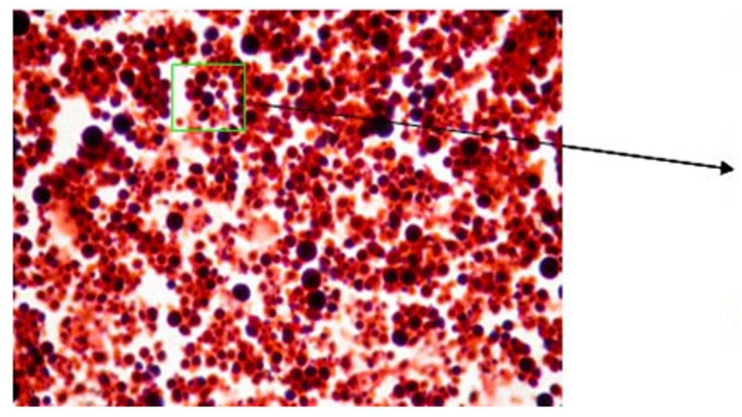

(c)

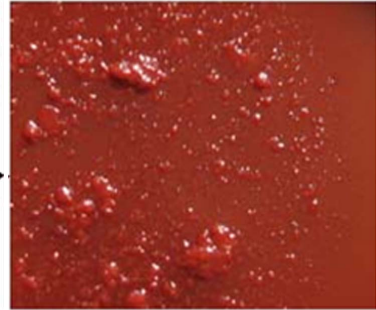

(b)

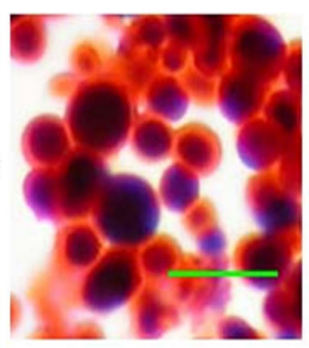

(d)

Figure 2. Colonies of normal blood flora on agar plate. (a) Colonies of blood microbiota on agar plate. Culture is performed for 30 days at $43^{\circ} \mathrm{C}$; (b) zoom in of the sector plate; (c) Gram stained single colony; (d) Several bacterial "dense body" forms of Gram positive, Gram negative and forms with Gram positive center and Gram negative coat could be observed. Green bar $10 \mu \mathrm{m}$. 
Several physicochemical characteristics were examined. Disruption of cells for $15 \mathrm{~min}$ with $0.1 \mathrm{~mm}$ glass beads with beat beater machine (Mini-beatbeater, Biospec Products), treatment in microwave oven at $850 \mathrm{~W}$ for $3 \mathrm{~min}$ and disintegration with ultrasound (MSE, UK) for $1 \mathrm{~h}$ performed on ice applying maximum power pulsations, i.e. $15 \mathrm{~s}$ of disintegration and $15 \mathrm{~s}$ at rest were without success. Evaluation of microbial cell lysis or disintegration was performed microscopically after Gram staining. On the slides intact "dense bodies" were observed. Increasing the time for disintegration with ultrasound for $2 \mathrm{~h}$ brought to unexpected finding. Cells observed with yeast like shape on light microscopy and on electron microscopy as "dense bodies" at different stages of maturation were disintegrated to single "elementary bodies" with streptococcal appearance (Figures 3(a)-(c)). We conclude that the "dense bodies" give origin to elementary structures of "primary cells" with the size of $0.1-0.2 \mu \mathrm{m}$. Integrity of the "dense body" cells in fresh cultures was tested by treatments with $4 \mathrm{M}$ guanidine thiocianate, $10 \% \mathrm{NaOH}, 10 \% \mathrm{KOH}, 10 \% \mathrm{CH}_{3} \mathrm{COOH}, 10 \% \mathrm{HCl}$ and $10 \% \mathrm{H}_{2} \mathrm{SO}_{4}$ for $30 \mathrm{~min}$ at $37^{\circ} \mathrm{C}, 0.5 \mathrm{M}$ EDTA for 4 days at room temperature and freeze-thawing

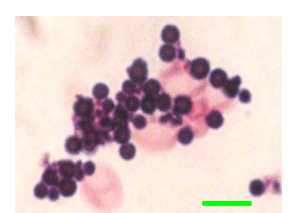

(a)
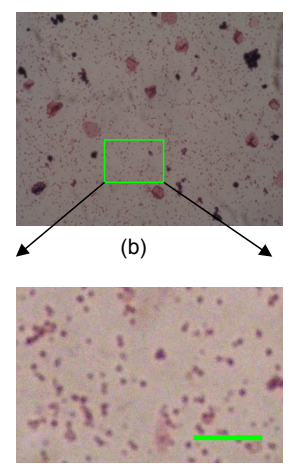

(c)

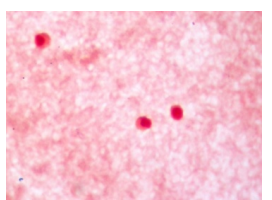

(d)

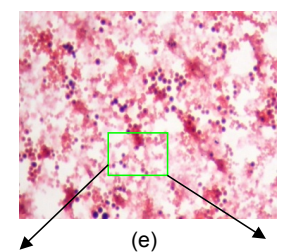

(e)

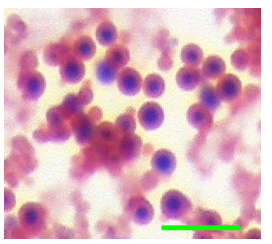

(f)

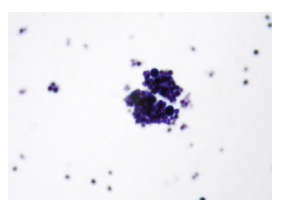

(g)

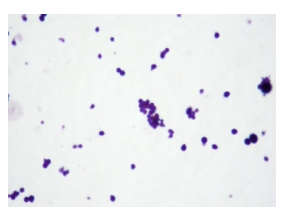

(h)

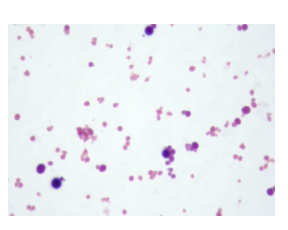

(i)

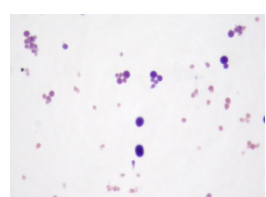

(j)

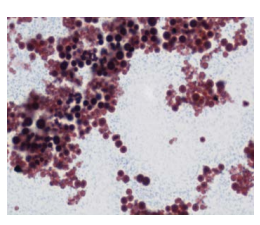

(k)

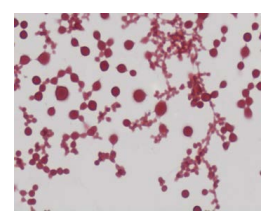

(I)

Figure 3. Resistance of the blood microbiota to physical and chemical treatments. (a) Gram stained microbial structures of blood microbiota grown on agar for 30 days at $43^{\circ} \mathrm{C}$; (b), (c) Disintegration with ultrasound at maximum power for one hour with pulsations of $30 \mathrm{~s}$ of ultrasound and $30 \mathrm{~s}$ of rest. After disintegration with ultrasound "dense bodies" significantly decrease in number. "Cells" with streptococcal appearance dominate on the microscopy field; (d) Non-cultured blood sample after gamma irradiation with 25 $+25 \mathrm{KGy}$ dose. Only blood cells could be observed. After the first irradiation with $25 \mathrm{KGy}$ growth was observed after 14 days of culture (not shown). Second dose of $25 \mathrm{KGy}$ was applied to the same sample; (e) $25+25 \mathrm{KGy}$ irradiated sample cultured for 14 days at $43^{\circ} \mathrm{C}$; (f) Sector zoom-in of the blood microbiota. Cells seem not affected by this extreme dose of irradiation; (g) Blood microbiota treated with $10 \% \mathrm{KOH}$; (h) with $10 \% \mathrm{NaOH}$; (i) with $10 \% \mathrm{CH}_{3} \mathrm{COOH}$ and (j) with $10 \% \mathrm{H}_{2} \mathrm{SO}_{4}$ for $30 \mathrm{~min}$ at $37^{\circ} \mathrm{C}$; (k) $0.5 \mathrm{M}$ EDTA for 4 days at room temperature and (l), freeze-thawing resistance in liquid nitrogen and $96^{\circ} \mathrm{C}$. Green bar $10 \mu \mathrm{m}$. 
resistance in liquid nitrogen and $96^{\circ} \mathrm{C}$. We observed that only Gram-positive microbial structures were seen after treatment with bases. The treatment with acids decreased the number of observed Gram-negative structures. Microbial cells were partially affected by these coarse treatments as observed on microscopy (Figures 3(g)-(k)).

Blood microbiota resisted to high dose of $25+25 \mathrm{kGy}$ gamma irradiation with ${ }^{60} \mathrm{Co}$ (Figures 3(d)-(f)). Growth of radioresistant blood microbiota was detected after two weeks. This is not surprising, because the majority of soil bacteria are killed by $20 \mathrm{kGy}$, however dose higher than $70 \mathrm{kGy}$ may be required to kill certain radio-resistant bacteria [19].

For DNA analysis we applied 16S rRNA gene targeted sequencing of V3-V4 hypervariable region on Illumina MiSeq (Illumina Inc., USA). DNA sequencing was performed at IMGM Laboratories GmbH (Martinsried, Germany). The resulting $2 \times 300$ bp reads were demultiplexed, quality controlled and merged into continuous reads. Further bioinformatics analysis including clustering, phylogenetic analysis and alpha and beta diversity calculation was performed with the CLC genomics workbench and its microbial genomics module. In order to correctly describe the microbial biodiversity on phylum and order level, four filtering steps were applyed: 1) only Operational Taxonomic Units (OTUs) exceeding similarity of $>97 \%$ on genus level were included in the analysis, 2) only OTUs exceeding a combined abundance among all tested samples of 100 were included, 3) OTU reads per sample below $1 \%$ abundance at order level were excluded and 4) exclusion of common sequence reads originating from exogenous DNA. The sources of these contaminants are reagents used in DNA extraction, culture medium, PCR reagents, and next-generation sequencing library preparation. The sequences, available after trimming and merging showed a high amount of high quality reads, suitable for downstream analysis. Among the cultured and non-cultured samples sequencing analysis identified rich biodiversity of OTUs similarity ( $>97 \%$ on genus level) with 47 bacterial orders belonging to 15 phyla and 39 fungi orders blonging to 2 phyla. The bacterial orders were predominantly of phylum Proteobacteria. Among non-cultured samples Proteobacteria were 93\%, Actinobacteria 2\%, Planctomycetes $2 \%$ and Firmicutes $2 \%$, while among cultured samples Proteobacteria were 46\%, Firmicutes 25\%, Actinobacteria 14\%, Bacteroidetes 6\%, Fusobacteria 3\% and Cyanobacteria 2\%. The fungi phyla Basidiomycota, Ascomycota and unidentified fungi were 64\%, 22\% and $14 \%$ respectively among the non-cultured samples, while among the cultured samples they were $27 \%, 31 \%$ and $41 \%$ respectively. We note, that some phyla are enriched by the applied resuscitation strategy according to the OTUs combined abundancy (Table 1). Other studies also identified Proteobacteria as predominant in the blood of healthy individuals [14], healthy broilers [16] and cats [17].

Our results demonstrate that isolation of the blood microbiota is technically affordable by conventional means. We identified, that in $100 \%$ of the blood 
Table 1. OTUs abundances of identified bacterial and fungi taxa among non-cultured and cultured blood samples.

\begin{tabular}{|c|c|c|}
\hline BACTERIA & $\begin{array}{c}\mathrm{N} \text { : of reads in } \\
\text { non-cultured samples (\%) }\end{array}$ & $\begin{array}{c}\mathrm{N}: \text { of reads in cultured } \\
\text { samples }(\%)\end{array}$ \\
\hline Phylum: Acidobacteria & $44(<1 \%)$ & $421(1 \%)$ \\
\hline Phylum: Actinobacteria & $820(2 \%)$ & $9754(14 \%)$ \\
\hline Phylum: Armatimonadetes & $422(1 \%)$ & $239(<1 \%)$ \\
\hline Phylum: Bacteroidetes & $73(<1 \%)$ & $4580(6 \%)$ \\
\hline Phylum: Chlamydiae & 0 & $129(<1 \%)$ \\
\hline Phylum: Chloroflexi & $5(<1 \%)$ & $99(<1 \%)$ \\
\hline Phylum: Cyanobacteria & $179(<1 \%)$ & $1652(2 \%)$ \\
\hline Phylum: Firmicutes & $1159(2 \%)$ & $17635(25 \%)$ \\
\hline Phylum: Fusobacteria & $30(<1 \%)$ & $2284(3 \%)$ \\
\hline Phylum: Planctomycetes & $730(2 \%)$ & $297(<1 \%)$ \\
\hline Phylum: Proteobacteria & $43138(93 \%)$ & $32272(46 \%)$ \\
\hline Phylum: Saccharibacteria & $7(<1 \%)$ & $432(1 \%)$ \\
\hline Phylum: Spirochaetae & 0 & $262(<1 \%)$ \\
\hline Phylum: SR1 (Absconditabacteria) & 0 & $250(<1 \%)$ \\
\hline Phylum: Synergistetes & 0 & $198(<1 \%)$ \\
\hline \multicolumn{3}{|l|}{ FUNGI } \\
\hline Fungi unidentified & $45118(14 \%)$ & $187419(41 \%)$ \\
\hline Phylum: Ascomycota & $70401(22 \%)$ & $142425(31 \%)$ \\
\hline Phylum: Basidiomycota & $204113(64 \%)$ & $122503(27 \%)$ \\
\hline
\end{tabular}

samples the blood microbiota resuscitate in $24-48$ hours under stress by culturing at $43^{\circ} \mathrm{C}$ in high concentrations of vitamin $\mathrm{K}$. In vitro we identified by microscopy that the most common mechanism of formation and reproduction of the "dense body" is by production in chains Gram negatively stained "primary cells", as previously described by Domingue (1977) [5], (1997) [7]. We observed loss of the Gram negative component of the "dense body" after treatment with $10 \% \mathrm{NaOH}$. Evidences for intraerythrocytic presence of normal blood microbiota were reported by Kalfin in 1997 [6]. We identified free circulating microbiota, "primary cells", by electron microscopy in blood samples processed within the first hour after collection (Figures $1(\mathrm{e})-(\mathrm{g})$ ). We suppose that the "electron dense body" is composed by Gram negative and Gram positive 'primary cells'. Currently applied approaches for biodiversity analysis of blood microbiota use microbe-derived DNA or RNA that may have crossed into the blood from the gut. The proposed by us culture resuscitation approach overcomes these limitations. We showed that blood DNAemia in healthy individuals is associated wtih the presence of culturable microbiota in the blood. To prove this, cell-free DNA circulating in the blood (DNAemia) was eliminated by 
applying a triple DNAse I treatment, centrifugation and washing of the cell

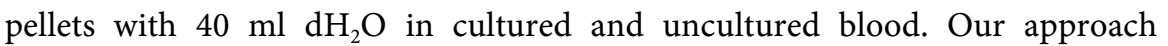
included the use of positive and negative controls. Contaminating sequences associated with reagents were excluded from analysis. Among cultured and non-cultured blood samples certain orders replicate well as demonstated by increase in OTUs abundance (Table 1). It could be concluded that the applied resuscitation culture conditions enrich specific orders while others remain unchanged. Moreover we predict that applying other growth conditions or media will change the composition of the cultured microbiota in the sample.

When grouping the samples according the blood type we identified significant differences. In non-cultured samples of individuals of blood type A we identified less number of reads but higher bacterial diversity at order level as compared to the other blood types (Table 2).

The identified taxa in the blood have been reported in previous studies of the blood microbiota in healthy individuals or animals. Mangul et al. (2016) identified 23 bacterial taxa at pylum level applying total RNA sequencing [16]. In a recent study Chen et al. (2017) showed that the uterus and the fallopian tubes, which are generally believed to be sterile, are home of rich microbiota indicative of a non-sterile environment [20].

Table 2. Bacterial blood microbiota according to the blood group. (a) Number of reads according to blood group and order in non-cultured blood samples; (b) Graphical distribution of the bacterial orders.

(a)

\begin{tabular}{ccccc}
\hline Taxonomy & Group A & Group B & Group AB & Group O \\
\hline p: Actinobacteria, o: Actinomycetales & 11 & 0 & 0 & 0 \\
p: Actinobacteria, o: Corynebacteriales & 64 & 0 & 95 & 346 \\
p: Actinobacteria, o: Micrococcales & 59 & 0 & 0 & 0 \\
p: Armatimonadetes, o: Fimbriimonadales & 0 & 131 & 0 & 238 \\
p: Bacteroidetes, o: Cytophagales & 32 & 0 & 0 & 0 \\
p: Cyanobacteria, o: Carya cathayensis & 34 & 0 & 0 & 0 \\
p: Cyanobacteria, o: uncultured bacterium & 31 & 0 & 0 & 0 \\
p: Firmicutes, o: Bacillales & 262 & 343 & 0 & 432 \\
p: Fusobacteria, o: Fusobacteriales & 29 & 0 & 0 & 0 \\
p: Planctomycetes, o: Planctomycetales & 0 & 216 & 113 & 275 \\
p: Proteobacteria, o: Rhizobiales & 36 & 9187 & 4309 & 7152 \\
p: Proteobacteria, o: Rhodobacterales & 117 & 668 & 0 & 1412 \\
p: Proteobacteria, o: Sphingomonadales & 119 & 6151 & 2495 & 10570 \\
p: Proteobacteria, o: Burkholderiales & 18 & 0 & 0 & 0 \\
p: Proteobacteria, o: Xanthomonadales & 33 & 0 & 0 & 0 \\
TOTAL & 845 & 16696 & 7012 & 20425
\end{tabular}


(b)

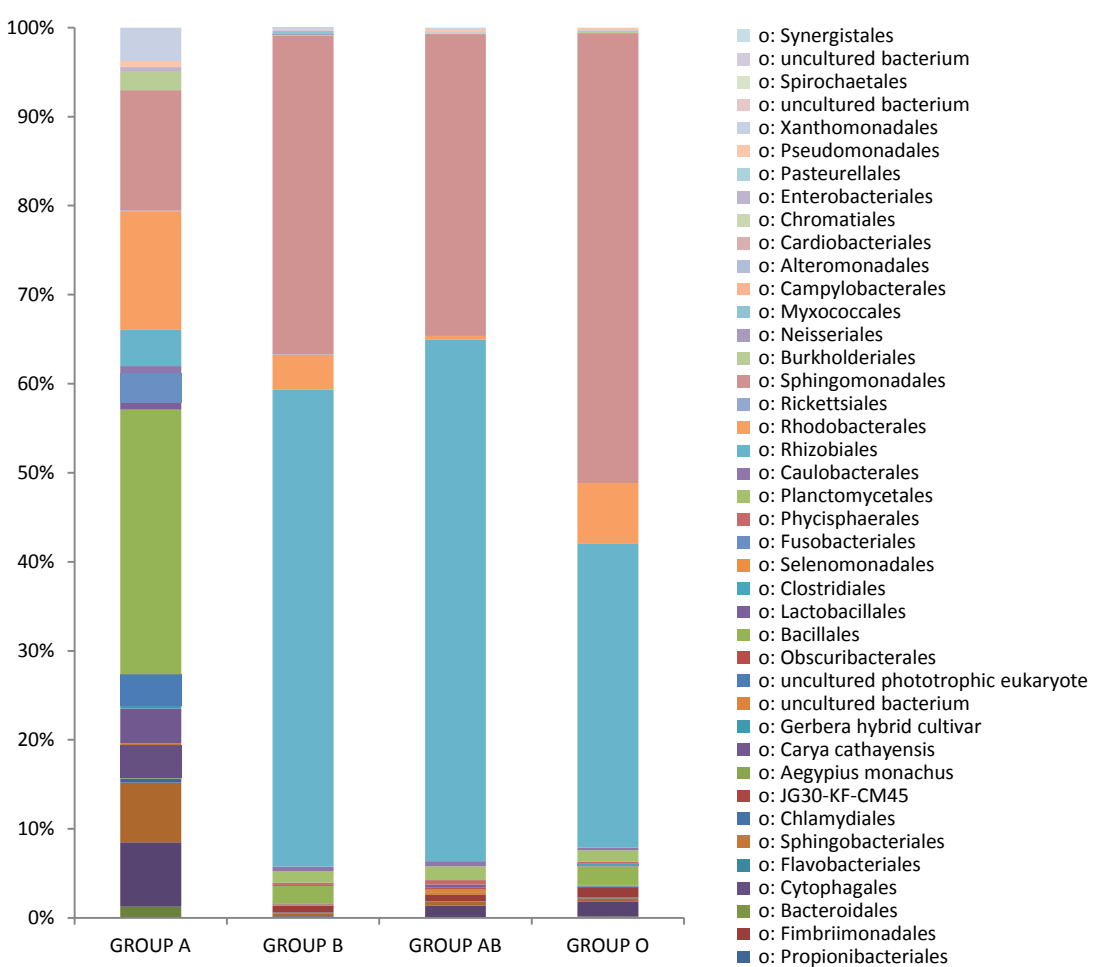

The bacterial and fungi composition of the blood microbiota is highly diverse and each individual or even blood group has its own specificities (Table 2, Table 3). The abundance of given OTUs is indicative for high prevalence and not contamination.

\section{Conclusion}

Here we demonstrated rich biodiversity of naturally occurring bacterial and fungal blood microbiota in healthy individuals. We developed a successful strategy for analysis of the blood microbiome by total microbial resuscitation by blood culturing, microscopy and NGS DNA sequencing. The total microbial resuscitation was proved by comparative analysis of cultured and non-cultured blood samples. Blood-group differences were identified among the bacterial microbiome composition. For the first time we identify rich fungiome in the blood. We showed that blood DNAemia in healthy individuals is also associated with the presence of culturable microbiota in the blood. Our results confirm that the blood of healthy humans is not as sterile as previously supposed by other authors [4]-[15] [20]. This work brings evidences, that the resident blood microbiota in healthy individuals should be considered non-pathogenic and a normal feature of the healthy blood. Furthermore, we anticipate our resuscitation strategy and sequencing approach to be a model for studying the numerous chronic diseases, such as obesity, diabetes, cardiac failure, liver diseases, hematologic disorders, neurodegenerative diseases and chronic infections, like latent tuberculosis. Beyond cataloguing the species of the blood environment, 
Table 3. Fungal blood microbiota according to blood group. (a) Abundance of OTU reads according to blood group and fungi orders in non-cultured blood samples; (b) Graphical distribution of the fungi orders.

(a)

\begin{tabular}{|c|c|c|c|c|}
\hline Taxonomy & Group A & Group B & Group $A B$ & Group O \\
\hline Fungi unidentified & 19213 & 3657 & 4598 & 17650 \\
\hline o:Caliciales & 2291 & 0 & 0 & 0 \\
\hline o:Capnodiales & 0 & 284 & 240 & 293 \\
\hline o:Chaetosphaeriales & 328 & 0 & 0 & 0 \\
\hline o:Diaporthales & 458 & 0 & 0 & 779 \\
\hline o:Dothideales & 1005 & 1377 & 0 & 6914 \\
\hline o:Erysiphales & 658 & 471 & 0 & 2227 \\
\hline o:Eurotiales & 0 & 1677 & 0 & 839 \\
\hline o:Helotiales & 1173 & 213 & 0 & 219 \\
\hline o:Hypocreales & 3339 & 0 & 14 & 881 \\
\hline o:Microascales & 0 & 420 & 0 & 0 \\
\hline o:Other & 3151 & 399 & 1015 & 4722 \\
\hline o:Pleosporales & 10624 & 1745 & 130 & 4741 \\
\hline $\mathrm{o}:$ Saccharomycetales & 330 & 970 & 2637 & 3492 \\
\hline o:Sordariales & 0 & 0 & 1925 & 0 \\
\hline o:Taphrinales & 1053 & 0 & 0 & 637 \\
\hline o:Xylariales & 0 & 358 & 383 & 5976 \\
\hline o:Agaricales & 0 & 1377 & 0 & 2818 \\
\hline $\mathrm{o}:$ Agaricostilbales & 536 & 0 & 0 & 0 \\
\hline o:Auriculariales & 2580 & 0 & 0 & 0 \\
\hline o:Boletales & 0 & 0 & 0 & 6289 \\
\hline o:Cantharellales & 0 & 0 & 0 & 556 \\
\hline o:Corticiales & 2355 & 0 & 0 & 1022 \\
\hline o:Cystobasidiales & 0 & 0 & 0 & 1433 \\
\hline o:Cystofilobasidiales & 1551 & 0 & 0 & 0 \\
\hline o:Filobasidiales & 2709 & 0 & 205 & 19742 \\
\hline o:Holtermanniales & 0 & 0 & 0 & 4102 \\
\hline o:Hymenochaetales & 0 & 2382 & 0 & 1982 \\
\hline o:Malasseziales & 1151 & 937 & 3730 & 0 \\
\hline o:Microstromatales & 2187 & 0 & 1207 & 0 \\
\hline o:Other & 11674 & 4115 & 3619 & 13801 \\
\hline o:Polyporales & 16349 & 4990 & 6562 & 14683 \\
\hline o:Pucciniales & 0 & 0 & 0 & 3968 \\
\hline o:Russulales & 1005 & 1654 & 280 & 3153 \\
\hline $\mathrm{o}:$ Sporidiobolales & 740 & 4869 & 2777 & 2249 \\
\hline o:Thelephorales & 0 & 1411 & 683 & 2523 \\
\hline o:Tremellales & 15209 & 15610 & 1411 & 7848 \\
\hline o:Ustilaginales & 0 & 0 & 0 & 2046 \\
\hline TOTAL & 101669 & 48916 & 31416 & 137585 \\
\hline
\end{tabular}


(b)

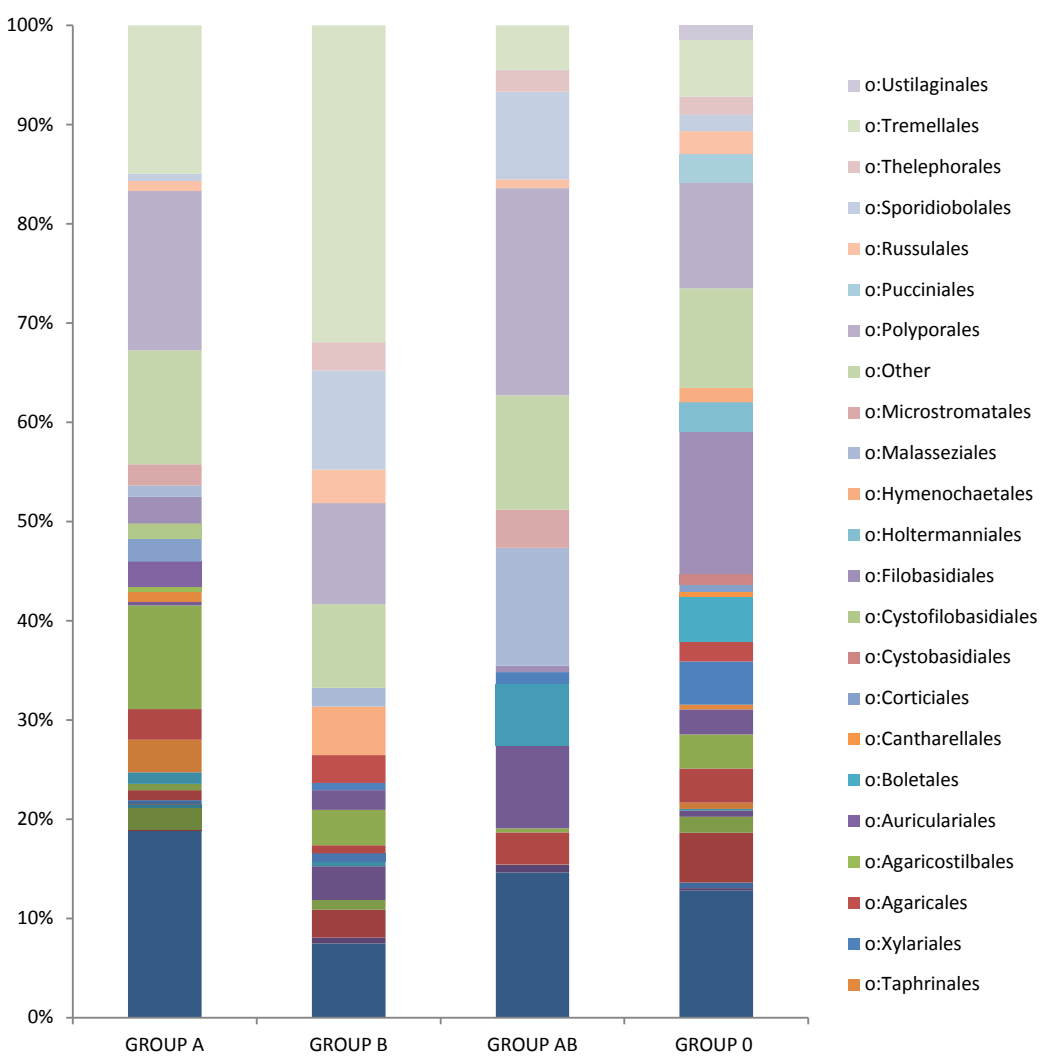

the blood microbiome field will focus on defining the mechanisms underpinning the interactions between microbes and host, mechanisms influencing the initiation and progression of diseases with a view towards personalization of patient's diagnosis and treatment.

\section{Acknowledgements}

This project has received funding from the National Science Fund of Bulgaria GA: ДН-01-4/16.12.2016.

\section{References}

[1] The HMP Consortium. (2012) A Framework for Human Microbiome Research. Nature, 486, 215-221. https://doi.org/10.1038/nature11209

[2] Gosiewski, T., Ludwig-Galezowska, A.H., Huminska, K., Sroka-Oleksiak, A., Radkowski, P., Salamon, D., et al. (2017) Comprehensive Detection and Identification of Bacterial DNA in the Blood of Patients with Sepsis and Healthy Volunteers Using Next-Generation Sequencing Method-The Observation of DNAemia. European Journal of Clinical Microbiology and Infectious Diseases, 36, 329-336. https://doi.org/10.1007/s10096-016-2805-7

[3] Kowarsky, M., Camunas-Soler, J., Kertesz, M., De Vlaminck, I., Koh, W., Pan, W., et al. (2017) Numerous Uncaracterized and Higly Divergent Microbes Which Colonize Humans Are Revealed by Circulating Cell-Free DNA. Proceedings of the National Academy of Sciences of United States of America, 114, 9623-9628. 
https://doi.org/10.1073/pnas.1707009114

[4] Tedeschi, G.G., Amici, D. and Paparelli, M. (1969) Incorporation of Nucleosides and Amino-Acids in Human Erythrocyte Suspensions: Possible Relation with a Diffuse Infection of Mycoplasmas or Bacteria in the L Form. Nature, 222, 1285-1286. https://doi.org/10.1038/2221285a0

[5] Domingue, G.J. and Schlegel, J.U. (1977) Novel Bacterial Structures in Human Blood: Cultural Isolation. Infection \& Immunity, 15, 621-627.

[6] Kalfin, E. (1997-1998) Resident Microbial Flora in Human Erythrocytes. Journal of Culture Collections, 2, 77-82.

[7] Domingue, G.J. and Woody, H.B. (1997) Bacterial Persistence and Expression of Disease. Clinical Microbiology Reviews, 10, 320-334.

[8] Nikkari, S., McLaughlin, I., Bi, W., Dodge, D., Relman, D. (2001) Does Blood of Healthy Subjects Contain Bacterial Ribosomal DNA? Journal of Clinical Microbiology, 39, 1956-1959. https://doi.org/10.1128/JCM.39.5.1956-1959.2001

[9] McLaughlin, R.W., Vali, H., Lau, P., Palfree, R., De Ciccio, A., Sirois, M., et al. (2002) Are There Naturally Occurring Pleomorphic Bacteria in the Blood of Healthy Humans? Journal of Clinical Microbiology, 40, 4771-4775. https://doi.org/10.1128/JCM.40.12.4771-4775.2002

[10] Moriyama, K., Ando, C., Tashiro, K., Kuhara, S., Okamura, S., Nakano, S., et al. (2008) PCR Detection of Bacterial 16S rRNA Gene in Human Blood. Microbiology and Immunology, 52, 375-382. https://doi.org/10.1111/j.1348-0421.2008.00048.x

[11] Markova, N., Slavchev, G. and Michailova, L. (2015) Presence of Mycobacterial L-Forms in Blood: Challenge of BCG Vaccination. Human Vaccines and Immunotherapeutics, 11, 1192-1200. https://doi.org/10.1080/21645515.2015.1016682

[12] Damgaard, C., Magnussen, K., Enevold, C. Nilsson, M., Tolker-Nielsen, T., Holmstrup, P. and Nielsen, C. (2015) Viable Bacteria Associated with Red Blood Cells and Plasma in Freshly Drawn Blood Donations. PloS ONE, 10, e0120826. https://doi.org/10.1371/journal.pone.0120826

[13] Dimova, T., Terzieva, A., Dierov, L., Dimitrova, V., Nikolov, A., Grozdanov, P. and Markova, N. (2017) Mother-to-Newborn Transmission of Mycobacterial L-Forms and V82 T-Cell Response in Placentobiome of BCG-Vaccinated Pregnant Women. Nature Scientific Reports, 7, 17366. https://doi.org/10.1038/s41598-017-17644-Z

[14] Païssé, S., Valle, C., Serfant, F., Courtney, M., Burcelin, R., Amar, L., et al. (2016) Comprehensive Description of Blood Microbiome from Healthy Donors Assessed by 16S Targeted Metagenomic Sequencing, Transfusion 56, 1138-1147. https://doi.org/10.1111/trf.13477

[15] Mangul, S., Loohuis, L., Ori, A., Jospin, G., Koslicki, L., Yang, H., et al. (2016) Total RNA Sequencing Reveals Microbial Communities in Human Blood and Disease Specific Effects. bioRxiv057570.

[16] Mandal, R., Jiang, T., Al-Rubaye, A., Rhoads, D., Wideman, R., Zhao, J., et al. (2016) An Investigation into Blood Microbiota and Its Potential Association with Bacterial Chondronecrosis with Osteomyelitis (BCO) in Broilers. Nature Scientific Reports, 6, Article No. 25882. https://doi.org/10.1038/srep25882

[17] Vientos-Plotts, A.I., Ericsson, A., Rindt, H., Grobman, M., Graham, A., Bishop, K., et al. (2017) Dynamic Changes of the Respiratory Microbiota and Its Relationship to Fecal and Blood Microbiota in Healthy Young Cats. PLoS ONE, 12, e0173818. https://doi.org/10.1371/journal.pone.0173818

[18] Kalfin, R., Filevski, G., Equestre, M., Nikolova, E. and Panaiotov, S. (2017) Dormant 
Fungi in the Blood Microbiome of Healthy Individuals. F1000Research, 6, 1168.

[19] McNamara, N., Black, H., Bresford, N. and Parekh, N. (2003) Effects of Acute Gamma Irradiation on Chemical, Physical and Biological Properties of Soils. Applied Soil Ecology, 24, 117-132. https://doi.org/10.1016/S0929-1393(03)00073-8

[20] Chen, C., Song,X., Wei, W., Zhong, H., Dai, J., Lan, Z., et al. (2017) The Microbiota Continuum along the Female Reproductive Tract and Its Relation to Uterine-Related Diseases. Nature Communications, 8, 875.

https://doi.org/10.1038/s41467-017-00901-0 